\title{
Cognitive Apprenticeship: Appropriate Surgical Education for Countries with Limited Resources
}

\author{
Lowell L Lewis ${ }^{1,2}$ and Nicholas A Kerna ${ }^{3,4 *}$ \\ ${ }^{1}$ Ministry of Health, Government of Montserrat, BWI \\ ${ }^{2}$ Montserrat Institute of Surgical Research and Education, BWI \\ ${ }^{3}$ Office of Research and Clinical Studies, Suriwongse Medical Center, Thailand \\ ${ }^{4}$ SMC Research, Thailand \\ *Corresponding author: Nicholas A Kerna, SMC Research, P.O. Box 1026, Silom Road, Bangkok, Thailand 10504, Email: drkerna@gmail.com
}

Submission: February 18, 2019; Published: February 22, 2019

\begin{abstract}
The role of surgical education in the prevention and management of surgical disorders remains an important topic for study. In the absence of sufficient resources, developing countries struggle to meet the growing demands for adequate surgical services. People living in remote and underdeveloped areas, lacking modern medical facilities, are at significant risk for increased morbidity and mortality. To ameliorate this dilemma requires the identification and implementation of educational programs and curricula that provide training and experience that can be provided in remote communities. Governments of countries with limited resources must identify, retain, and develop methods of surgical practice and education that will result in adequate care to surgical patients, particularly those living in remote communities who do not have access to current standards of care. The primary objective of this paper is to document and share educational and management activities that may have a constructive impact on lessening the global burden of diseases and conditions requiring surgery; also, in promoting a surgical care approach that is affordable and can be applied in various ways to obtain favorable results in countries with limited resources.
\end{abstract}

Keywords: Apprenticeship; Articulation; Coaching; Cognitive; Exploration, Modeling; Performance-based; Reflection; Scaffolding; Surgical training

Abbreviations: CA: Cognitive Apprenticeship; CLR: Countries with Limited Resources

\section{Introduction}

According to Collin, Brown, \& Newman (1989), "Cognitive apprenticeship focuses on 'learning-through-guided-experience' on cognitive and metacognitive skills and processes" instead of the physically particular craft or trade that is the focus of traditional apprenticeships [1]. Cognitive apprenticeship (CA) is intended to enable apprentices to learn strategies and skills in the context of their application to real problems within a culture focused on and defined by expert practice. Required media of instruction in CA include experts, peers, instructors, and apprenticeshipbased computer systems. CA encourages reflection on differences between novice and expert performance. In CA, tasks are sequenced to reflect the changing demands of learning: increasing complexity, increasing diversity, and global before local skills. The facilitator's role in CA involves modeling, coaching, and scaffolding to help students acquire an integrated set of cognitive and metacognitive skills through processes of observation and guided and supported practice [2]. CA instructional strategies include the following:

a) Modeling that involves experts carrying out a task so that students observe and build a conceptual model of the processes required to accomplish the task;

b) Coaching that consists of observing student performance and offering hints, scaffolding, feedback, modeling, reminders, and new tasks;

c) Scaffolding provided by the teacher to help students carry out a task;

d) Articulation that allows students to express their knowledge, reasoning, and problem-solving processes in a domain;

e) Reflection that enables students to compare their problemsolving processes with those an expert, peer, and an internal cognitive model of expertise;

f) Exploration sets general goals for students and encourages students to focus on particular subgoals of interest to them;

g) and performance-based assessment [1-3] (Figure 1).

CA assumes that people learn from others through observation, imitation, and modeling. In CA, experts (usually teachers or mentors) demonstrate a task explicitly. New students or novices build a conceptual model of the task at hand. According to Collins \& Stevens (1982):

Students to articulate their knowledge, reasoning or problemsolving process in a domain: a process known as "scaffolding". 
Figure 1: Performance-based assessment (online).

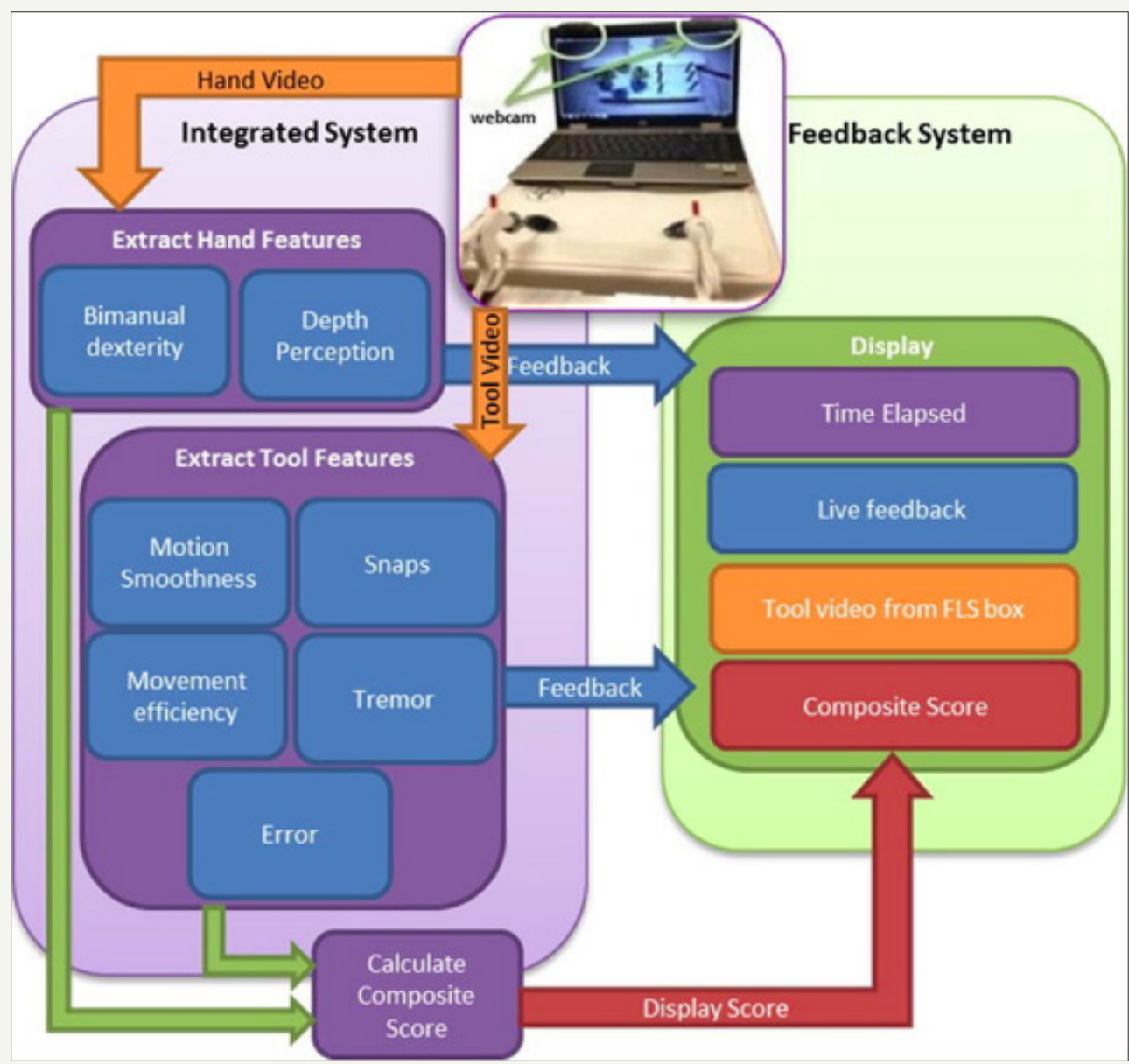

Note: Reproduced from Islam Li smit \& Patel. (2016). Affordable, web-based training and evaluation tool. Journal of Biomedical Informatics. Volume 59, February 2016, Pages 102-144 [4].

They compare their problem-solving processes with those of an expert or another student, and ultimately develop an internal cognitive model of expertise and reflect on and analyze their performances to understand and improve their behavior towards that of the expert: a process known as "reflection". Finally, the mentor or teacher slowly withdraws the supports and allows the student to frame problems within the domain and try to solve these problems: a process known as "exploration" [5].

\section{Discussion}

The governments of many countries with limited resources (CLR) are expected by their constituents to provide high quality and up-to-date surgical services. Yet, in practice, most populations of CLR experience inadequate access to basic and essential health care services [6]. Thus, there is a push by governments of CLR to close the gap between modern medical technology and procedures and the elemental practices typical of CLR. However, these governments should proceed with caution and due diligence, particularly regarding surgical procedures. The adaptation of modern technologies, such as laparoscopic and robotic surgical services, for CLR is desirable, but must not replace the traditional surgical procedures and skills and clinical approaches which remain the most affordable and, therefore, the most appropriate services for the vast majority of the world's population [7].

Choy, Kitto, Adu-Aryee, \& Okrainec (2013) provided clear guidelines for overcoming the "Barriers to the uptake of laparoscopic surgery in lower-middle-income countries". They identified three overarching barriers:

1) organizational structure for funding laparoscopic procedures,

2) hierarchical nature of the local surgical culture,

3) and expertise and skills associated with a change in practice [8].

The 1904 William Halsted models of surgical residency programs adopted worldwide have been modified in some developing countries to include primary examination, rural surgical posting, and submission of a dissertation for final certification [9]. Ajao and Alao (2016) concluded: "The innovations introduced into surgical residency training programs in developing countries are 
the result of the misconception of what surgical residency training programs entail" [9]. Other researchers in this field, such as Blankstein, Dakurah, Bagan, \& Hodaie (2011), supported a model of structured online surgical education and concluded that structured online courses in neurosurgery "allow for dynamic feedback and delivery of educational material in a 'time-locked' curriculum". They also added that curriculum design should create courses that "fill specific needs or identify educational objectives" [10].

Special attention is required for coping with the "Large Burden" demands for coping with orthopaedic diseases in developing countries [11]. A significant increase in the number of service providers and orthopaedic training programs is required to address these conditions [12].

International collaboration has a role in meeting this deficiency as reported by Cameron, Rambaran, Dee, Sharma, \& Taylor (2010) in "International surgery: the development of postgraduate surgical training in Guyana". They concluded: "Guyana has proven that, with visiting faculty assistance, it can mount postgraduate training suitable to national needs and will provide a career path to encourage its doctors to remain and serve their country" [14]. Medical student clerkships and electives contribute through "supervised, independent student learning and global health service that is integrated into a traditional surgical clerkship" [15].

The consortium approach to surgical education was illustrated by Cook et al. (2015) [16]. They identified seven areas of educational need:

1. Formal didactic

2. Increased clinical mentorship

3. Longer-term alliance presence

4. Equitable distribution of teaching time

5. Improved coordination

6. Language skills

7. Reciprocal exchange rotations at US hospitals [16].

Similar missions to Haiti and Brazil have provided surgical services to underserved populations by training local doctors and building local sustainability $[17,18]$. Also, several institutions have reported successful global health initiatives for surgical residents and faculty which were effectively integrated into academic surgical residency programs through relationships with training programs in low-income countries [6].

The role of the internet in promoting new surgical skills in developing countries has been recognized. Medical students in Nigeria, participating in a cross-sectional survey by a structured self-administered questionnaire, provided responses which led to the following conclusion by Ekenze, Okafor, Ekenze, Nwosu, \& Ezepue (2017):

In our setting, a substantial number of undergraduate medical students are familiar with the use of internet tools for learning and believe that the tools may have utility in surgical education.
However, to further consolidate and enhance the learning experience, it may be useful to integrate the learning modality with the traditional model if teaching through a well thought out curriculum modification [19].

Udwadia and Sen (2008) reflected on fifty years of surgical education in India, as follows:

Surgical training has to be tailored to the specific needs of each country. "Surgery is a humanitarian science. The thrust of surgical training in India should be, and hopefully will be, not just to be on par with the developed world, but more important, to ensure good surgical care to all Indians, in all places [20]".

CA, when applied to traditional methods of surgical education as well as the newer methods utilizing digital technology for telesimulation training, is the approach most likely to reduce the unmet surgical needs and burdens of CLRs and communities which are remote and have limited resources $[21,22]$.

\section{Conclusion}

In their rightful and duty-bound quest to stay current in the latest surgical techniques, governments of countries, especially those with limited resources, should look for innovative, acceptable, and economical ways to keep their surgical training up-to-date. For traditional and especially advanced surgical techniques, cognitive apprenticeship can be utilized as an effective and particularly economical means to train surgeons in the latest surgical techniques.

Cognitive apprenticeship is an accepted learning method that has been recognized for decades. It utilizes the interactions of experts, peers, instructors, and apprenticeship-based computer systems $[1,2]$. It involves modeling, coaching, scaffolding, articulation, reflection, and exploration; and includes performance-based assessment $[3,4]$. In short, proponents of cognitive apprenticeship have shown that students, in this case surgical students, learn from mentors, experts, and peers through observation, imitation, and modeling.

Governments of countries with limited resources should remain mindful to keep and practice their traditional procedures as these are the procedures that most of the patients will be administered, particularly in outlying areas.

\section{Conflict of Interest Statement}

The authors declare that this paper was written in the absence of any commercial or financial relationship that could be construed as a potential conflict of interest.

\section{Supplementary Note}

This paper is based on prior published research: Lewis, LL (2017) APPROPRIATE SURGICAL EDUCATION FOR COUNTRIES WITH LIMITED RESOURCES Cognitive Apprenticeship: The Why, When and How of Surgical Practice (published Master Thesis in Experimental Surgery - Surgery Education). Chapter I \& 2. McGill University Digitool Library StreamGate. (C)2017 Lowell L Lewis. 


\section{Acknowledgment}

The first author wishes to acknowledge Dr. Michael L Hoover, Associate Professor, Department of Educational and Counseling Psychology (ECP), McGill University, Member of the Research Advisory Committee, for his instruction in EDPE664 (Expertise Reasoning and Problem-solving) and his inspiration, guidance, and mentoring during thesis development for the first author's Master of Science in Experimental Surgery - Surgical Education. Also, the authors wish to recognize the Montserrat Institute of Surgical Research and Education, Montserrat, British West Indies.

\section{References}

1. Collins A, Brown JS, Newman SE (1987) Cognitive apprenticeship: Teaching the craft of reading, writing and mathematics (Technical Report No. 403). BBN Laboratories, ${ }^{\circ}$ Cambridge, MA. Centre for the Study of Reading, University of Illinois, USA.

2. Collins A, Brown JS, Newman SE (1988) Cognitive apprenticeship thinking. The Journal of Philosophy for Children 8(1): 2-10.

3. Kerna NA (2018) Communication among physicians and allied healthcare associates the role and rank of medical terminology in surgery, public health and the history of medicine. SM Prev Med Public Health 2(2): 1020 .

4. Islam G, Kahol K, Li B, Smith M, Patel V (2016) Affordable, web-based surgical skill training and evaluation tool. Journal of Biomedical Informatics 59: 102-114

5. David L (2012) Cognitive apprenticeship in learning theories.

6. Abdool Carrim AT, Veller MG (2010) Vascular surgical education in a medium income country. European Journal of Vascular \& Endovascular Surgery 39(Suppl 1): S22-24

7. Afuwape $O 0$ (2012) An affordable laparoscopic surgery trainer for trainees in poor resource settings. West African Journal of Medicine 31(1): 63-65.

8. Choy I, Kitto S, Adu-Aryee N, Okrainec A (2013) Barriers to the uptake of laparoscopic surgery in a lower-middle-income country. Surg Endosc 27(11): 4009-4015.

9. Ajao OG, Alao A (2016) Surgical residency training in developing countries: west African college of surgeons as a case study. Journal of the National Medical Association 108(3): 173-179.

10. Blankstein U, Dakurah T, Bagan M, Hodaie M (2011) Structured online neurosurgical education as a novel method of education delivery in the developing world. World Neurosurgery 76(3-4): 224-230.
11. Andreatta P, Perosky J, Klotz J, Gamble C, Ankobea F et al. (2013) Pilot study outcomes from a resource-limited setting for a low-cost training program for laparoscopic surgical skills. International Journal of Gynaecology \& Obstetrics 125(2): 186-188.

12. Brouillette MA, Kaiser SP, Konadu P, Kumah Ametepey RA, Aidoo AJ et al. (2014) Orthopedic surgery in the developing world: workforce and operative volumes in Ghana compared to those in the United States. World Journal of Surgery 38(4): 849-857.

13. Cameron BH, Rambaran M, Deen P Sharma DP, Taylor RH (2010) International surgery: the development of postgraduate surgical training in Guyana. Canadian Journal of Surgery 53(1): 11-16.

14. Cadotte DW, Sedney C, Djimbaye H, Bernstein M (2014) A qualitative assessment of the benefits and challenges of international neurosurgical teaching collaboration in Ethiopia. World Neurosurgery 82(6): 980-986.

15. Chin Quee A, White L, Leeds I, MacLeod J, Master VA (2011) Medical student surgery elective in rural Haiti: a novel approach to satisfying clerkship requirements while providing surgical care to an underserved population. World Journal of Surgery 35(4): 739-744.

16. Cook M, Howard BM, Yu A, Grey D, Hoffman PB et al. (2015) A consortium approach to surgical education in a developing country: educational needs assessment. JAMA Surgery 150(11): 1074-1078.

17. Damas E, Norcéide C, Zephyr Y, Williams KL, Renouf T, et al. (2016) Development of a sustainable simulator and simulation program for laparoscopic skills training in Haiti. Cureus 8(6): e632.

18. De Rosa A, Meyer ALM, Seabra A, Sorge A, Hack J, et al. (2016) An international surgical collaboration: humanitarian surgery in Brazil. Hernia 20(4): 553-557.

19. Ekenze SO, Okafor CI, Ekenze OS, Nwosu JN, Ezepue UF (2017) The value of internet tools in undergraduate surgical education: perspective of medical students in a developing country. World Journal of Surgery 41(3): 672-680.

20. Udwadia TE, Sen G (2008) Surgical training in India. World Journal of Surgery 32(10): 2150-2155.

21. Okrainec A, Henao O, Azzie G (2010) Telesimulation: an effective method for teaching the fundamentals of laparoscopic surgery in resourcerestricted countries. Surgical Endoscopy 24(2): 417- 422.

22. Plummer JM, Roberts PO, Leake PA, Mitchell DI (2001) Surgical care in Jamaica in the laparoendoscopic era: challenges and future prospects for developing nations. Permanente Journal 15(1): 57-61.
Creative Commons Attribution 4.0

International License

For possible submissions Click Here

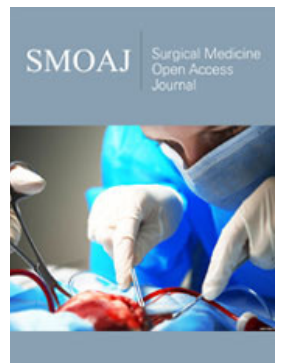

\section{Surgicall Medicine Open Access Journal}

\section{Benefits of Publishing with us}

- High-level peer review and editorial services

- Freely accessible online immediately upon publication

- Authors retain the copyright to their work

- Licensing it under a Creative Commons license

- Visibility through different online platforms 